\title{
Comparison of Excitation of Acoustic-Electromagnetic Wave in Piezoelecric Crystal and Crystal with Potential of Deformation
}

\author{
S. Koshevaya, V. Grimalsky, Y. Kotsarenko, J. Escobedo-Alatorre \\ Autonomous University of Morelos State, CIICAp, Cuernavaca, Mexico \\ Email: svetlana@uaem.mx
}

Received 29 July 2015; accepted 19 October 2015; published 22 October 2015

Copyright @ 2015 by authors and Scientific Research Publishing Inc.

This work is licensed under the Creative Commons Attribution International License (CC BY). http://creativecommons.org/licenses/by/4.0/

\section{(c) (i) Open Access}

\begin{abstract}
In this article, the comparison of excitation in high frequencies of acoustic-electromagnetic wave in piezoelecric crystal and crystal with potential of deformation GaAs is investigating. Possible mechanisms of coupling different hybrid waves are the piezoeffect and the deformation potential. As a model it is analyzing a film of crystal places between two symmetrical substrates with the other materials without an acoustic contact. This film includes 2D electron gas with a high negative differential conductivity and uniform initial distribution of electrons. The hybrid acoustic-electromagnetic wave and hybrid space charge wave interact. Amplification of space charge wave takes place due to negative differential conductivity in GaAs. This amplification of space charge waves is causing the amplification of acoustic-electromagnetic wave. It is to show that the symmetric modes, emerging as transverse ones, interact more effectively with the space charge waves. Another important result is the following: at the frequencies $f \approx 10 \mathrm{GHz}$, the excitation efficiency of acoustic-electromagnetic wave with transverse displacement due to piezoeffect is more effective, but at higher frequencies, the deformation potential is dominating.
\end{abstract}

\section{Keywords}

Piezoelectric Crystal, Deformation Potential, Acoustic-Electromagnetic and Space Charge Waves

\section{Introduction}

The amplification of the acoustic-electromagnetic waves is very important problem [1]. A possible solution of

How to cite this paper: Koshevaya, S., Grimalsky, V., Kotsarenko, Y. and Escobedo-Alatorre, J. (2015) Comparison of Excitation of Acoustic-Electromagnetic Wave in Piezoelecric Crystal and Crystal with Potential of Deformation. Journal of Electromagnetic Analysis and Applications, 7, 259-264. http://dx.doi.org/10.4236/jemaa.2015.710027 
this problem is the resonant coupling of acoustic waves with the microwave electric field of space charge waves (SCW) in materials possessing negative differential conductivity (NDC) like GaAs [2] [3]. Namely, under propagation in the bias electric field higher than the critical value for observing negative differential conductivity (NDC), the SCW is subject to amplification, and its microwave electric field can achieve high values. Due to piezoeffect or deformation potential, this microwave electric field excites hypersonic acoustic-electromagnetic waves (AEW). In [2] [3], it is demonstrated that this excitation has a resonant character with respect to the frequency and the thickness of the GaAs film. The critical value of bias electric field in GaAs is $E_{c}=3.5 \mathrm{kV} / \mathrm{cm}$ that it is good for analysis of amplification in different mechanisms like piezoeffect or deformation potential so as other materials have very big critical field like crystal InP and GaN.

The present paper is focused on theoretical research of excitation of acoustic-electromagnetic waves (AEW) in GaAs films of a sub-micron thickness placed between non-piezoelectric substrates. The spatial increments of SCW due to NDC have been calculated for two mechanisms like piezoeffect and deformation potential. An amplification of SCW is possible for frequencies from $10 \mathrm{GHz}$ for case of piezoeffect. For deformation potential the frequencies range increase from $10 \mathrm{GHz}$ to $30 \mathrm{GHz}$, so the mechanism of deformation potential is more useful in high frequencies.

The thin GaAs film plays the role in a transducer of acoustic electromagnetic waves. The amplification has a resonant character, and the intensities of energy may reach the values of $10 \mathrm{~W} / \mathrm{cm}^{2}$. The realization of the good amplification has showed that usefulness of hybrid waves in high frequencies range more $f \approx 10 \mathrm{GHz}$ is very important for application in communication, medicine and control systems. The analysis of basic mechanisms of amplification is given in this article. The resonance excitation and amplification of acoustic-electromagnetic wave with space mode in structure of GaAs is analyzing for two cases of piezoeffect and deformation potential, but the amplification of high frequencies are realizing only in case of deformation potential.

\section{Model of Amplification Due to Piezoeffect}

Let us to consider a thin film of n-GaAs located between two mediums, the substrates are from material without piezoeffect, and the transversal wave passed along $z$ axis. The component of displacement $U$ is in direction of the $y$ axis. The film consists of a 2D gas with a high negative differential mobility. The value $L$ is the length of the element (along axis OZ), $h$ is the thickness of film (see Figure 1). This film has a substrates with depth $d$. The hybrid wave is decreasing in the substrate, then, all its energy is in the very thin film with 2D electron beam. The effective size of the waveguide is describing by the parameter $2 H=2(h+d / 2), d$ is the depth of substrate.

Let us to analyze the simplest transversal in displacements $u$ mode using elastic theory equation with the piezoeffect [1]. It is necessary to use the variable electric field $E=-\frac{\partial \varphi}{\partial z}$ (in direction of axes Z) determined by potential $\varphi$,

$$
\frac{1}{s^{2}} \frac{\partial^{2} u}{\partial t^{2}}=\Delta u+\Gamma \Delta \frac{\partial u}{\partial t}-\frac{2 \beta}{\rho s^{2}} \frac{\partial^{2} \varphi}{\partial x \partial z}
$$
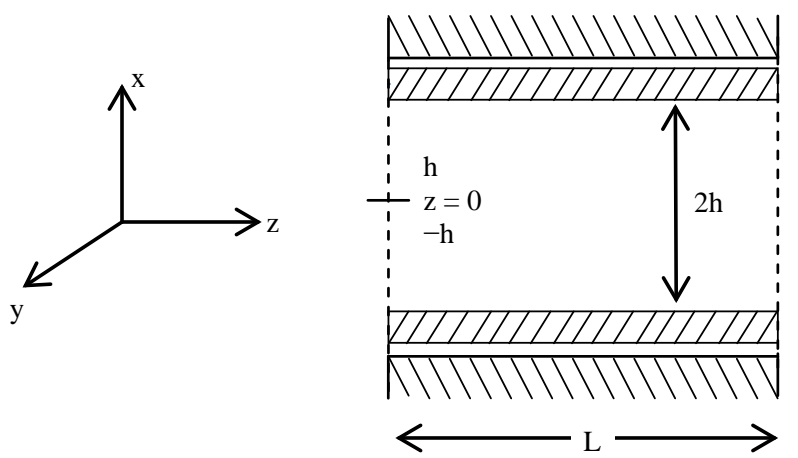

Figure 1. Geometry of waveguide for computing simulation of amplification acoustic-electromagnetic wave where $2 h$ is the transversal size of guide in $x$ direction and $L$ is the length of the guide. In direction $y$ the model of guide has size going to infinitive value. 


$$
\begin{aligned}
& -\Delta \varphi+\frac{\beta}{\varepsilon_{0} \varepsilon} \frac{\partial^{2} \varphi}{\partial x \partial y}=0 \\
& {\left[\frac{\partial u}{\partial x}+-\frac{\beta}{\rho s^{2}} \cdot \frac{\partial \varphi}{\partial z} \mid x=+,-h / 2\right]=0,}
\end{aligned}
$$

where $s \approx 3 \times 10^{5} \mathrm{~cm} / \mathrm{s}$ is the sound velocity, $\beta$ is the module of coefficient of piezoeffect, $\rho$ is the density parameter, $\varphi$ is the potential determined the electric field $\operatorname{grad} \varphi=\overrightarrow{\boldsymbol{E}}$ [1]-[5].

The boundary conditions are in (Equation 1(c)). In the case of free very thin film it is $\frac{h}{d / 2+h} \prec \prec 1$. In this case it is necessary to consider the viscosity coefficient $\Gamma$ for the simulation. The wave process shows that the amplification realizes if the synchronism between the acoustic-electromagnetic wave with transversal displacement mode and the space charge wave [5],

$$
k_{0} \cong \sqrt{\frac{\omega_{0}^{2}}{s^{2}}-\frac{(2 m+1)^{2} \pi^{2}}{4 H^{2}}}=\frac{\omega_{0}}{v_{0}}
$$

where $\omega_{0}$ is the frequency of synchronism, $s, v_{0}$ is sound and space charges waves velocities, respectively; index $m(1,2,3, \cdots) \succ \succ 1), H$ must have also an optimal value.

Resonance condition $\cos g H \cong 0$ must hold true, where

$$
g=\frac{\omega_{c r}}{s}=\frac{(2 m+1) \pi}{2 H}
$$

Parameters $g$ and $\omega_{c r}$ are given by

$$
g=\frac{\omega_{c r}}{s}=\frac{(2 m+1) \pi}{2 H}
$$

and

$$
\omega_{c r}^{2}=\frac{\pi^{2} s^{2}}{4 H^{2}}(2 m+1)^{2}
$$

These conditions for the amplification are better for the symmetric modes if the displacement

$$
u_{n} \propto B \cdot \sin g x \cdot e^{i(\omega t-k z)}, \phi_{n} \propto \frac{i k \pi}{g} \cdot B \cdot \cos g x \cdot \mathrm{e}^{i(\omega t-k z)} .
$$

Boundary conditions are taken in case of the absent of the acoustic contact of the film with the environment (Equation 1(c)).

In the simulation it is to use Equation (1) and the resonance condition (2). It is necessary to take into account the 2D electron gas of n-GaAs thin film and the equations for slow changing amplitudes of acoustic-electromagnetic hybrid wave with potential $\varphi$. The computer modeling shows the effective amplification of waves in the presence of the negative differential mobility (conductivity). The following parameters have been chosen $n_{0} \approx$ $10^{15} \mathrm{~cm}^{-3}$, the electron concentration in the film, $v_{0} \approx 2 \times 10^{7} \mathrm{~cm} / \mathrm{s}$, the electron velocity, $L=0.1 \mathrm{~cm}$, the length of the film $H=1 \mu \mathrm{m}$. The intensity of wave can reach value of $10 \mathrm{~W} / \mathrm{cm}^{2}$ at microwave frequencies $\omega=5 \times 10^{10}$ $2 \times 10^{11} \mathrm{~s}^{-1}$. In the Figures 2-4, the amplification of the acoustic-electromagnetic wave in microwave range is to present.

Traditional construction for realization of acoustic-electromagnetic amplification is in Figure 3. This construction is for high frequencies $f \approx 10^{11} \mathrm{~Hz}$ ranges too.

Additionally, in simulations it is considering the electric variable field distribution in input antenna like Gauss impulse.

$$
E^{\sim}(z, y, t)=E_{10} \exp \left(-\left(\left(z-z_{1}\right) / z_{0}\right)^{2}-\left(\left(y-y_{1}\right) / y_{0}\right)^{2}\right) \times \exp \left(-\left(\left(t-t_{1}\right) / t_{0}\right)^{2}\right)
$$




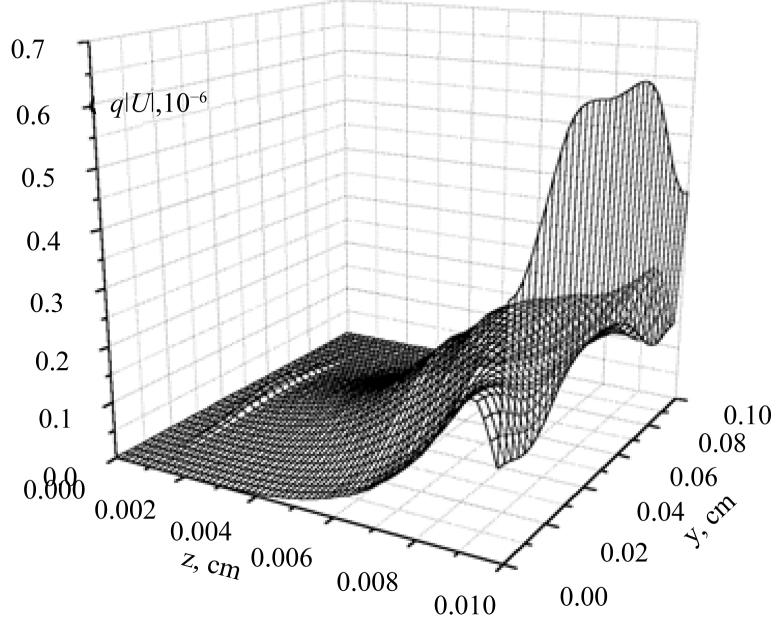

Figure 2. Distribution of the amplitude of acoustic deformation $q|U|$ along the film in direction $z$ for time $t=4$ ns. The resonant excitation is given at frequency $\omega=10^{11} \mathrm{~s}^{-1}$ of the hybrid acoustic-electromagnetic mode, and the size of the waveguide is $H=1 \mu \mathrm{m}$.

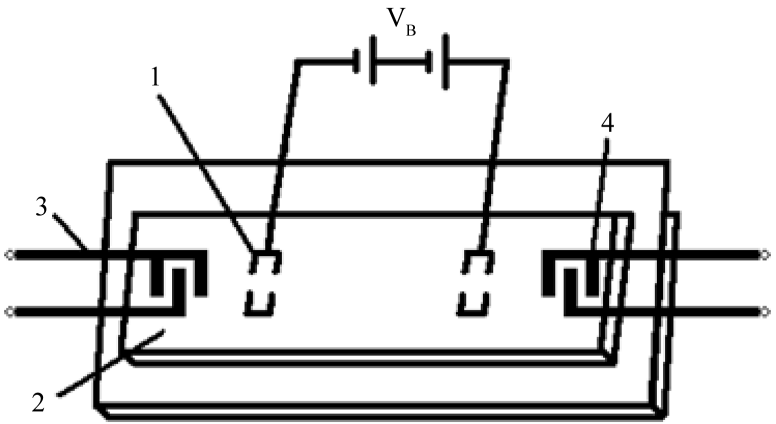

Figure 3. Traditional structure for exiting of acoustic-electromagnetic waves. It is necessary to prepare the cathode and anode 1 , and the input 3 and the output 4 antennas; the film of $n-G a A s$ is in $\mathrm{i}-\mathrm{GaAs}$ substrate.

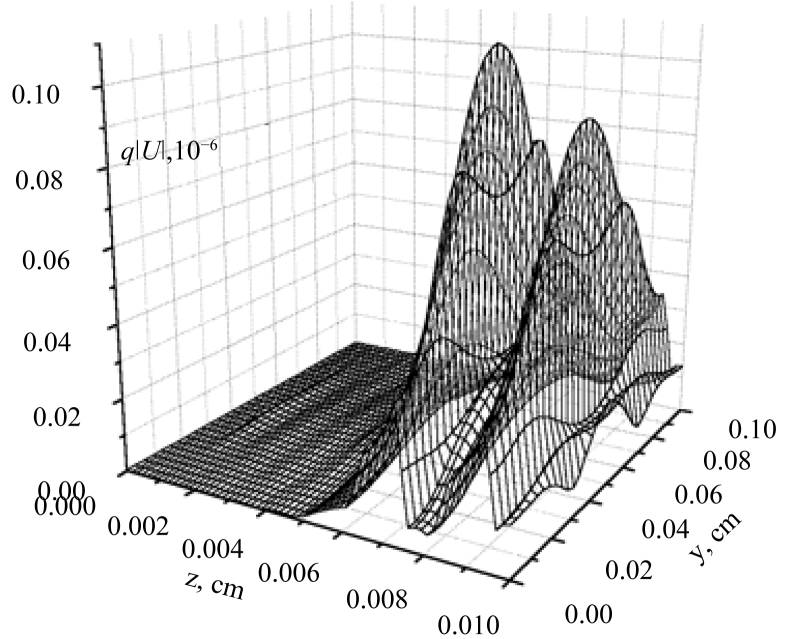

Figure 4. Distribution of the displacements deformation amplitude along the film in direction $z$ at time $t=4$ ns with a size of wave guide $H=0.1 \mu \mathrm{m}$. 
Electric variable field distribution in input antenna has parameters: $z_{1}=0.001 \mathrm{~cm}, z_{0}=0.0001 \mathrm{~cm} ; y_{1}=0.05 \mathrm{~cm}$, $y_{0}=0.02 \mathrm{~cm} ; t_{1}=2.5 \mathrm{~ns}, t_{0}=1.25 \mathrm{~ns} ; E_{10}=200 \mathrm{~V} / \mathrm{cm}$ and $E_{B}=4.56 \mathrm{kV} / \mathrm{cm}$, the drift field, $n_{0}=10^{11} \mathrm{~cm}^{-2}$, the $2 \mathrm{D}$ concentration of electron gas, and $q$, the transverse wave number (index $m$ equal 5 ) of the acoustic-electromagnetic mode.

Excitation of the acoustic-electromagnetic wave due to deformation potential is in the Lamb's acoustic mode [1], which possess longitudinal component of mechanic displacement, has the electric field too and interacts with space charge waves due to the deformation potential. The equation for the elastic displacement components of the Lamb's wave [1] $u=\left(u_{1}, 0, u_{3}\right)$ takes a form.

$$
\begin{aligned}
& \rho \frac{\partial^{2} u_{1}}{\partial t^{2}}=c_{11} \frac{\partial^{2} u_{1}}{\partial x^{2}}+c_{44} \frac{\partial^{2} u_{1}}{\partial z^{2}}+\left(c_{12}+c_{44}\right) \frac{\partial^{2} u_{3}}{\partial x \partial z}+G \Delta \frac{\partial u_{1}}{\partial t}+\frac{1}{3} \Xi_{u}^{L}\left(\frac{\partial n^{L}}{\partial y}+\frac{\partial n^{L}}{\partial z}\right), \\
& \rho \frac{\partial^{2} u_{3}}{\partial t^{2}}=c_{11} \frac{\partial^{2} u_{3}}{\partial z^{2}}+c_{44} \frac{\partial^{2} u_{3}}{\partial x^{2}}+G \Delta \frac{\partial u_{3}}{\partial t}+\left(c_{12}+c_{44}\right) \frac{\partial^{2} u_{1}}{\partial x \partial z}+\Xi_{d}^{\Gamma} \frac{\partial n^{\Gamma}}{\partial z}+\left(\Xi_{d}^{L}+\frac{1}{3} \Xi_{u}^{L}\right) \frac{\partial n^{L}}{\partial z}+\frac{1}{3} \Xi_{u}^{L} \frac{\partial n^{L}}{\partial y}
\end{aligned}
$$

Here $\Xi_{d, u}^{\Gamma, L}$ are the components of tensor of deformation potential; $c_{i j}$ are the elastic constants [1] [5] [6], the term $G \Delta \partial u / \partial t$ is due to dissipation, as in the Equation (3). Note that the partial concentrations $n^{L}, n^{\Gamma}$ for the valleys $\Gamma, L$ are present in the Equations (3).

As in the case of piezoactive transverse modes, the effective excitation of acoustic-electromagnetic waves with displacement like in Equations (3) takes place near cut-off frequency. It is shown that the symmetric modes emerging as transverse ones interact more effectively with the space charge waves. A distribution of the displacements near cut-off is

$$
u_{1} \approx \sin \left(\frac{\omega}{s_{l}} x\right) \exp (i(\omega t-k z)) ; u_{3} \approx 0 ; \frac{\omega}{s_{l}} \approx \frac{\pi}{2 h}(2 n+1), n=0,1,2, \cdots,
$$

here $s_{l} \approx 5.5 \times 10^{5} \mathrm{~cm} / \mathrm{s}$ is the longitudinal sound velocity in GaAs [7].

The computer modeling in the base of Equations (3) and (4) is the same like for case the piezoeffect. This modeling has demonstrated that the distributions in the plane of the film of excited acoustic-electromagnetic waves are very similar to those obtained for piezoeffect. But it is shown $i$ that the efficiency of excitation due to deformation potential becomes dominating at higher frequencies $f>30 \mathrm{GHz}$.

\section{Conclusion}

The resonant interaction of space charge waves with acoustic-electromagnetic waves in thin GaAs films can be used for effective in microwave wave range. At the frequencies $f<30 \mathrm{GHz}$ efficiency of excitation of acousticelectromagnetic due to piezoeffect is more effective, but at higher frequencies, deformation potential is dominating. An intensity of acoustic-electromagnetic waves can reach $10 \mathrm{~W} / \mathrm{cm}^{2}$ at microwave frequencies range $f=$ $10 \mathrm{GHz} \div 30 \mathrm{GHz}$. To increase a frequency of excited wave to millimeter range, it is possible to choose the resonant excitation of acoustic-electromagnetic waves at the second harmonic of the input signal.

\section{Acknowledgements}

Authors thank SEP-CONACyT for the support of our work.

\section{References}

[1] Dieulesaint, E. and Royer, D. (2000) Elastic Waves in Solids. Springer, New York.

[2] Grimalsky, V., Gutierrez-D., E., Garcia-B., A. and Koshevaya, S. (2004) Resonant Excitation of Microwave Acoustic Modes in n-GaAs Film. Proceedings of International. Conference on Microelectronics ICM-2004, Tunis, 6-8 December 2004, 72-75. http://dx.doi.org/10.1109/icm.2004.1434209

[3] Grimalsky, V., Gutierrez-D., E., Garcia-B., A. and Koshevaya, S. (2006) Resonant Excitation of Microwave Acoustic Modes in n-GaAs. Microelectronics Journal, 37, 395-403. http://dx.doi.org/10.1016/j.mejo.2005.06.003

[4] Mitin, V.V., Kochelap, V.A. and Stroscio, A. (1999) Quantum Heterostructures: Microelectronics and Optoelectronics. Cambridge University Press, Cambridge. 
[5] Shur, M. (1989) GaAs Devices and Circuits. Plenum Press, New York.

[6] Carnez, B. (1980) Modeling of Submicrometer Gate FET Including Effect of Nonstationary Dynamics. Journal of Applied Physics, 51, 784-790.

[7] Koshevaya, S.V., Grimalsky, V.V., Garcia-B., A. and Díaz-A., M.F. (2006) Amplification of Hypersonic by GaAs Crystals. Ukrainian Journal of Physics, 51, 594-598. 